\title{
Positioning of the Tibial Tunnel After Single-Bundle ACL Primary Reconstruction on 3D CT scans: A New Method
}

\author{
Paul Cremer, Ph.D., Adrien Peltier, Ph.D., Laurent Maubisson, Ph.D., \\ Philippe Neyret, M.D., Ph.D., Sébastien Lustig, M.D., Ph.D., and Elvire Servien, M.D., Ph.D.
}

\begin{abstract}
Purpose: To assess intra-articular tunnel aperture positioning after primary anterior cruciate ligament (ACL) reconstruction with either the reference standard method or the intercondylar area method in a single center using 3dimensional (3D) computed tomography (CT) scans and to evaluate the intra-articular position of the tibial tunnel relative to the ACL footprint. Methods: 3D CT scans were performed after 120 single-bundle primary ACL reconstruction cases. The center of the tibial tunnel aperture and the center of the ACL footprint were referenced on axial views of the tibial plateau in the anteroposterior (AP) and mediolateral (ML) planes according to a centimetric grid system including the whole plateau (reference standard). This was compared with a grid system based on intercondylar area bony anatomy. The posterior aspect of intertubercular fossa, anterior aspect of the tibial plateau, medial intercondylar ridge, and crossing point between lateral intercondylar ridge and posterior margin were used as landmarks to define the grid. Results: According to the reference standard method, the center of the tibial tunnel aperture was positioned $0.57 \pm 2.62$ $\mathrm{mm}$ more posterior and $0.67 \pm 1.55 \mathrm{~mm}$ more medial than the center of the footprint. According to the intercondylar area method, the center of the tibial tunnel aperture was positioned $1.32 \pm 2.74 \mathrm{~mm}$ more posterior and $0.66 \pm 1.56 \mathrm{~mm}$ more medial than the center of the footprint. The position difference between the center of the tunnel aperture and the center of the footprint were statistically correlated for both grids, with $r=-0.887, P<.001$ for AP positioning and $r=0.615, P<.001$ for ML positioning. Conclusion: This intercondylar area method using arthroscopic landmarks can be used to assess tunnel placement on 3D CT scans after ACL reconstruction. Level of Evidence: III, retrospective comparative study.
\end{abstract}

D uring anterior cruciate ligament (ACL) reconstruction, accurate positioning of bony tunnels is highly correlated to stability control, and thus to clinical outcome. Conversely, improper positioning is associated with high rates of failure. ${ }^{1-3} \mathrm{~A}$ greater understanding of the complex anatomy of the native ACL has led to a greater emphasis on anatomic ACL

From the Hôpital Privé Saint-Martin, Pessac, France (P.C.), the Hôpital Privé Pays de Savoie, Annemasse, France (A.P.), IAE de L'université de Tours, Tours, France (L.M.), and the Centre Albert Trillat, Hôpital de la Croix Rousse, Lyon, France (P.N., S.L., E.S.).

The authors report that they have no conflicts of interest in the authorship and publication of this article. Full ICMJE author disclosure forms are available for this article online, as supplementary material.

Received October 16, 2019; accepted July 16, 2020.

Address correspondence to Paul Cremer, Ph.D., Hôpital privé Saint Martin, Allée des Tulipes, 33600 Pessac, France.E-mail:paul.cremer@hotmail.fr

(C) 2020 THE AUTHORS. Published by Elsevier Inc. on behalf of the Arthroscopy Association of North America. This is an open access article under the CC BY-NC-ND license (http://creativecommons.org/licenses/by-nc-nd/4.0/). 2666-061X/191247

https://doi.org/10.1016/j.asmr.2020.07.011 reconstruction in lieu of isometric reconstruction. ${ }^{4}$ Single-bundle ACL reconstructions target the center of the native ACL footprint for the aperture of the tibial tunnel. ${ }^{3,5,6}$ The footprint has been described in detail in anatomic $^{7-9}$ and radiological ${ }^{10,11}$ studies. It is located on the anterior aspect of the tibial plateau on the intercondylar area.

3-Dimensional (3D) computed tomography (CT) scan is now commonly used to assess appropriate tunnel position, and this frequent use leads to technical improvement for individual surgeons. ${ }^{12}$ Its reliability is better than that of standard radiography or magnetic resonance imaging (MRI), and the effective radiation dose is reported relatively low. ${ }^{13}$

Difficulties and pitfalls during ACL reconstruction are due to the reproducibility of the native anatomy rather than to the drilling of the tunnel itself. Therefore, the surgeon must be able to accurately assess the positioning of the drilled tunnels and compare it to the position of the ACL footprint. Several grids described in the literature can be used to evaluate the anteroposterior (AP) and mediolateral (ML) positioning of either the center of 
the tibial tunnel aperture or of the native footprint. ${ }^{14,15}$ Although, in these studies, the position of the tibial footprint is expressed as a percentage of global tibial plateau AP and ML length, the tibial insertion of the ACL is located in the intercondylar area, and only landmarks in this region are identifiable arthroscopically. Plateau depth and width remain unknown and of low interest during the procedure. Thus it seems to be interesting to pass over the lateral and medial plateau and focus on the intercondylar area to assess the positioning of tibial tunnels after ACL single-bundle reconstruction. For this purpose, and like previous studies concerning femoral tunnel positioning, ${ }^{16-18}$ we established a measuring grid based on identifiable landmarks in the intercondylar region.

In this article, we assess intra-articular tunnel aperture positioning after primary ACL reconstruction with either the reference standard method or the intercondylar area method in a single center using 3D CT scans and to evaluate the intra-articular position of the tibial tunnel relative to the ACL footprint. Our hypothesis was that an intercondylar area method, using margins visible on 3D CT scans and during arthroscopic procedures, permits an assessment of the position of the center of the tibial tunnel relative to the center of the footprint.

\section{Methods}

As part of the standard practice in our university department, CT scans were obtained within 6 weeks postoperatively between January 2014 and July 2015 to evaluate tibial and femoral tunnel positioning after primary single-bundle ACL reconstruction. CT scans of 120 primary ACL reconstructions were retrospectively reviewed. Exclusion criteria were age $<18$ years (15), secondary reconstructions (6), CT scan performed by another imaging service (118), and multiligament reconstructions (4). Age, sex, and all clinical data were systematically listed during pre- and postoperative clinical assessment and were available in patient files.

Each CT scan was performed by the same imaging service. The scanner was a CR-Brilliance CT (cut thickness, $0.67 \mathrm{~mm}$; increment, $0.33 \mathrm{~mm}$ ). The images were processed using Centricity ${ }^{\circledR}$ software (GE Medical Systems, Chicago, IL; 2006).

The ethics committee approved this study (CAL no. 2016-037). Our medical practice was not modified for this study. No additional examinations have been performed. CT scans have been performed routinely for all patients with ACL reconstruction since 2012.

\section{D Reconstruction}

The standardized 3D reconstruction protocol is inspired by Amis and Jakob's ${ }^{14}$ description of tibial tunnel positioning and has been used in several studies. $^{10,13,18}$ A 3D bony reconstruction of the knee was obtained. It was visualized by its medial aspect, and both femoral condyles were superimposed to obtain a strict lateral view of the knee. ${ }^{19}$ Femur and patella were manually erased. A line parallel to the medial articular tibial surface and crossing the posterior angle of the tibial plateau was drawn, and the whole tibia situated underneath was also manually erased. The reconstructed plateau was manually manipulated to obtain a superior view. The center of the tibial tunnel was marked with a dot.

Bony landmarks used to locate the ACL footprint were the anterior ridge, the anterior border of the intertubercular fossae, the medial intercondylar ridge, and the medial border of the lateral groove (respectively the anterior, the posterior, the medial, and the lateral boundary). ${ }^{20,21}$ An ellipse was drawn within these landmarks to model the native ACL footprint. The center of this ellipse was also marked with a dot, corresponding to the aimed position for the center of the tibial tunnel (Fig 1).

Reconstructions were all performed by a single surgeon $(\mathrm{PC})$.

\section{Evaluation of the Tunnels}

\section{Reference Standard Technique}

A rectangular grid was positioned in line with the anterior, posterior, medial, and lateral cortices on the axial view. Measurements included the size of the plateau, the position of the center of the ACL footprint, and the position of the center of the tunnel. The measurements were performed in millimeters from the anterior and from the medial border to the marked dot (Fig 2).

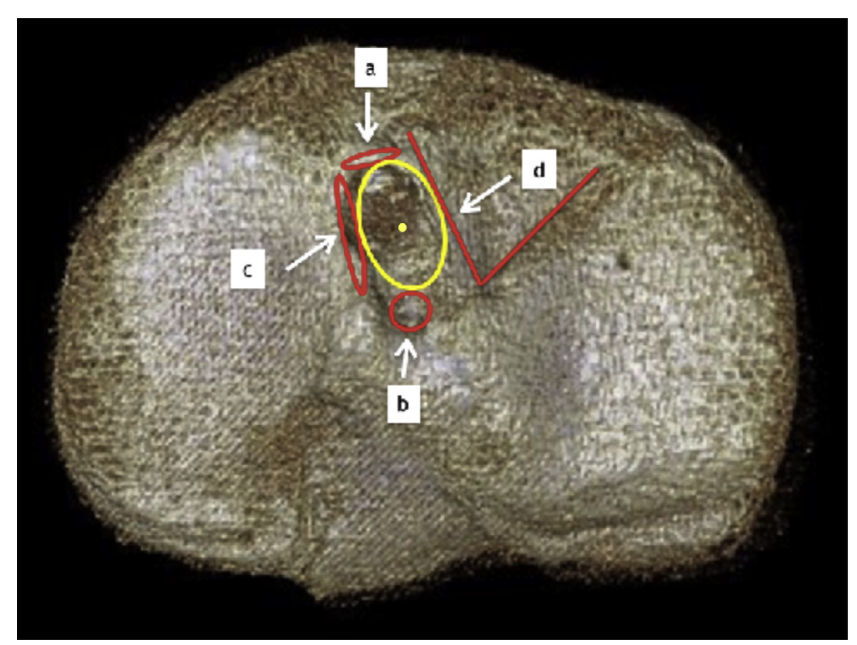

Fig 1. 3-Dimensional computed tomography scan reconstruction of a right tibial plateau axial view. Bony landmarks for ACL footprint and its center (yellow circle and yellow dot) are represented $(\mathrm{a}$, anterior ridge; $\mathrm{b}$, intertubercular fossae; $\mathrm{c}$, medial intercondylar ridge; $d$, medial border of the lateral groove). 


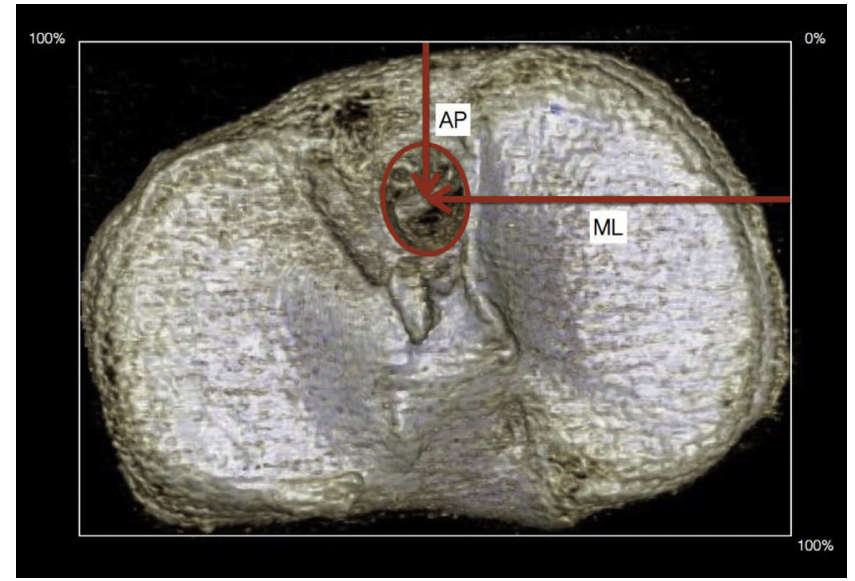

Fig 2. 3-Dimensional computed tomography scan reconstruction of a left tibial plateau axial view, with reference standard grid superimposed. The position of the center of the tunnel was measured from the anterior border and from the medial border in millimeters (17 and $40 \mathrm{~mm}$, respectively, in this example), then expressed as a percentage of the total anteroposterior and mediolateral dimension of the grid $(53$ and $76 \mathrm{~mm}$, respectively; therefore $32 \%$ and $52 \%$ in this example).

\section{Intercondylar Area Method}

A rectangular grid was positioned in line with the posterior margin of the intertubercular fossa and with the medial intercondylar ridge (Fig 3, A and B). Its anterior and lateral boundary were aligned respectively to the anterior tibial cortex, and with the intersection point between the posterior margin and the lateral intercondylar ridge (Fig 3, C and D). Measurements included the size of the grid, the position of the center of the ACL footprint, and the position of the center of the tunnel. The measurements were performed in millimeters from the posterior and from the medial border to the marked dot.

Measurements were performed by a single observer (P.C.), once on each grid.

\section{Statistical Analysis}

Pearson correlation coefficients were calculated using the AP depth and ML width of each grid as independent values, with the different measurements from the ideal and drilled tunnel as dependent values. These correlations were taken to be statistically significant for $P<.05$.

In a secondary analysis, the drilled tunnel was compared to the ideal tunnel according to both methods. This analysis was carried out independently for AP, ML and both AP and ML measurements. A value $<2 \mathrm{~mm}$ between the center of the ideal tunnel compared to the drilled tunnel was considered accurate. Accuracy rate was compared between the 2 methods using chi-squared test.

\section{Results}

We included 120 3D CT scans reconstructions. Patient characteristics are summarized in Table 1.

\section{Measurements With Reference Standard Method}

The mean AP and ML length of the grid (meaning the true AP and ML length of the plateau) were 53.9 \pm 4.0 and $77.5 \pm 5.7 \mathrm{~mm}$, respectively. The center of the aperture of the tunnel was located $21.1 \pm 3.8 \mathrm{~mm}$ from the anterior margin $(39.1 \%$ of AP length) and 36.4 $\pm 3.5 \mathrm{~mm}$ from the medial margin of the grid $(47.0 \%$ of ML length). The center of the footprint was located $20.5 \pm 2.4 \mathrm{~mm}$ from the anterior margin $(38 \%$ of $\mathrm{AP}$ length) and $37.1 \pm 3.4 \mathrm{~mm}$ from the medial margin of the grid (47.9\% of ML length). Thus the center of the aperture of the tunnel was located $0.57 \pm 2.62 \mathrm{~mm}$ more posterior and $0.67 \pm 1.55 \mathrm{~mm}$ more medial than the center of the footprint (Table 2).

\section{Measurements With the Intercondylar Area Method}

The mean AP and ML length of the grid were $29.5 \pm 3.0$ and $9.4 \pm 2.1 \mathrm{~mm}$, respectively. The center of the aperture of the tunnel was located $8.8 \pm 3.2 \mathrm{~mm}$ from the posterior margin $(29.8 \%$ of posteroanterior [PA] length) and $3.4 \pm 1.8 \mathrm{~mm}$ from the medial margin of the grid $(36.1 \%$ of ML length). The center of the footprint was located $10.1 \pm 1.5 \mathrm{~mm}$ from the posterior margin (34.2\% of PA length) and $4.1 \pm 0.63 \mathrm{~mm}$ from the medial margin of the grid (46.6\% of ML length). Thus the center of the aperture of the tunnel was located $1.32 \pm 2.74 \mathrm{~mm}$ more posterior and $0.66 \pm 1.56$ $\mathrm{mm}$ more medial than the center of the footprint (Table 3).

The position differences between the center of the tunnel aperture and the center of the footprint were statistically correlated for both grids, with $r=-0.887, P$ $<.001$ for AP positioning and $r=0.615, P<.001$ for ML positioning.

\section{Accuracy of Tunnel Positioning (Secondary Analysis)}

Table 4 shows the accuracy of the tunnel positioning relative to the ACL footprint according to both methods. For the reference standard and for the intercondylar area method, respectively, the tunnel was considered accurate in $77(62.2 \%)$ versus $61(50.8 \%)$ cases in the AP plane $(P<.001), 104(86.7 \%)$ versus 97 $(80.8 \%)$ cases in the ML plane $(P<.001)$, and 66 $(55 \%)$ versus $47(39.2 \%)$ in both planes $(P<.001)$.

\section{Discussion}

Our results confirm that an intercondylar area method, using margins visible on 3D CT scans and during arthroscopic procedures, permits an assessment 

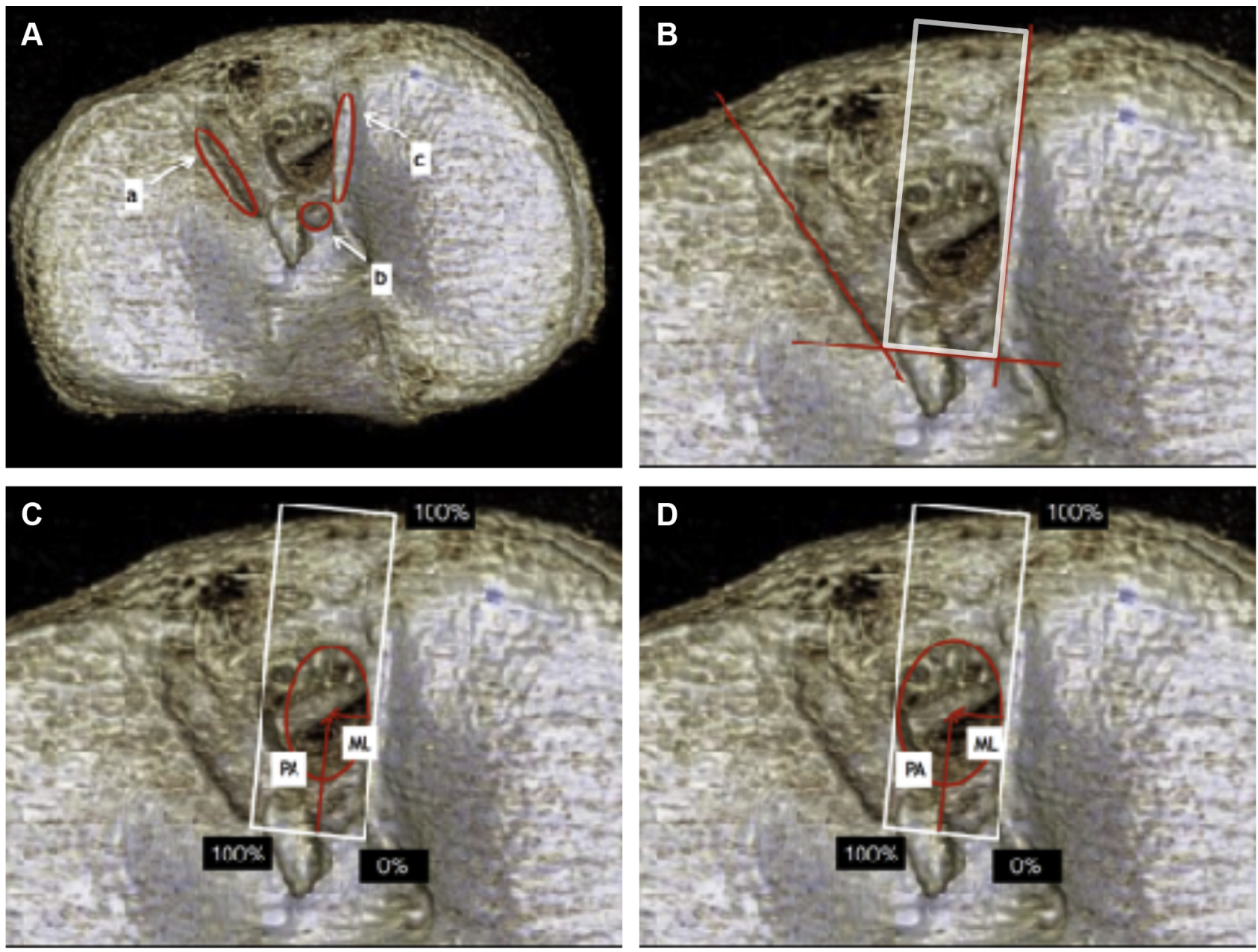

Fig 3. Measurement according to the intercondylar area method on a left knee. (A) Bony landmarks for grid placement (a, lateral intercondylar ridge; b, intertubercular fossa; c, medial intercondylar ridge. (B) Materializing the boundaries of the rectangular grid, aligned on the medial intercondylar ridge, the posterior border of the intertubercular fossa, the anterior tibial cortex, and the intersection point between the posterior margin of the grid and the lateral intercondylar ridge. (C) Center of the ACL footprint. (D) Center of the tunnel.

of the position of the center of tibial tunnel relative to the center of the footprint.

Our results regarding the location of the ACL footprint are in agreement with previous studies that used the same measurement technique. Parkinson et al. ${ }^{10}$ found on 3D CT scans of tibial plateau that the center of the footprint was located 39\% AP and 48\% ML. Sadoghi et al. ${ }^{22}$ found in a cadaveric study that the center of the footprint was located $41 \%$ AP and 49\% ML. Kongcharoensombat et al. $^{8}$ found that the center of the footprint was located $40.3 \%$ AP in another cadaveric study. Our estimate of the position of the footprint on 3D CT scans using the bony landmarks located in the intercondylar area, which can be made difficult by the presence of the tunnel, therefore seems reliable.

Concerning the intercondylar area method, we decided to measure the distance from posterior to anterior in the AP axis, in contrast to the reference standard method, which measures from anterior to posterior, because bony landmarks that are visible on 3D CT scans and also during arthroscopy, particularly the intercondylar ridge, are posterior. The anterior

Table 1. Patient and surgical data

\begin{tabular}{lc}
\hline \multicolumn{1}{c}{ Item } & Value \\
\hline Right/left & $65 / 55$ \\
Male/female & $75 / 45$ \\
Age at surgery $(\mathrm{y})$ & $33.33(13.6$ to 60.0$)$ \\
Graft & \\
$\quad$ Hamstring & 15 \\
Patellar tendon & 103 \\
Quadriceps tendon & 2 \\
Tunnel diameter $(\mathrm{mm})$ & \\
6 & 1 \\
7 & 3 \\
8 & 10 \\
9 & 98 \\
10 & 7 \\
11 & 1 \\
\hline Data are $\mathrm{n}$ or median (range)
\end{tabular}


Table 2. Comparison of position (in millimeters) between the center of tunnel and the center of the footprint according to reference standard method

\begin{tabular}{lccc}
\hline \multicolumn{1}{c}{ Position } & \multicolumn{3}{c}{$\begin{array}{c}\text { Standard } \\
\text { Deviation }\end{array}$} \\
\hline Grid (plateau) AP length & 53.9 & 4.0 & Range \\
Grid (plateau) ML width & 77.5 & 5.7 & 62.5 to 61.5 \\
Center of tunnel, AP & 21.1 & 3.8 & 12 to 30 \\
Center of tunnel, ML & 36.4 & 3.5 & 28 to 45 \\
Center of footprint, AP & 20.5 & 2.4 & 15.5 to 28 \\
Center of footprint, ML & 37.1 & 3.4 & 28 to 45 \\
Tunnel AP gap & 0.57 & 2.6 & -10 to 6.5 \\
Tunnel ML gap & -0.67 & 1.6 & -5 to 4 \\
\hline
\end{tabular}

Distances measured from anterior to posterior and from medial to lateral.

Abbreviations: AP, anteroposterior; ML, mediolateral.

aspect of the tibial plateau is variable in anatomy; therefore it is less reliable.

The intercondylar area method has several advantages over the reference standard technique. First, the reconstruction technique is easier to perform: reconstruct the knee in $3 \mathrm{D}$, subtract the femur and the patella, get a superior view, and add the measurement grid. There is no need to erase a part of the tibia.

Our secondary analysis shows that the positioning accuracy of the tunnels is different between the 2 methods. This is not surprising, since the grids are not necessarily oriented in the same direction. The positioning accuracy according to the intercondylar area method could better reflect reality, since it uses anatomic landmarks located in the intercondylar area visible during arthroscopy. The surgeon is more likely to adjust the tunnel positioning along these landmarks that allow building the intercondylar area grid rather than along the AP and ML axis of the reference standard grid. Indeed, the limits of the tibial plateau, which allow for the construction of the reference standard grid, are neither visible nor estimable during arthroscopy. The interest of the intercondylar area method is that it focuses on bony landmarks which are visible during arthroscopy and on 3D CT scans, ${ }^{23}$ allowing the surgeon to assess the positioning of tunnels in relation to concrete anatomic landmarks seen during the procedure.

Some studies have attempted to use 2D CT scans to evaluate the positioning of the tibial tunnel, but the tibial plateau is not completely flat, and the anatomic bony landmarks are not visible in the same plane and on a single cut of a CT scan. This geometry explains the limit of 2D CT imaging and the interest for 3D CT scans. ${ }^{13,24,25}$ Grasso et al. ${ }^{26}$ proposed an MRI protocol for routine quantitative assessment of tunnel position in ACL reconstruction, but MRI is not easily accessible.

Although the ACL remnant is key in positioning the tibial tunnel, ${ }^{9}$ several arthroscopic landmarks have been described in the literature to help to place the tibial tunnel in a reproducible manner whether a remnant is visible or not (during secondary reconstruction for example). The lateral border of the medial tibial eminence (described as "lateral border of medial tibial spine" $^{27}$ or "ridge at the lateral border of the medial tibial condyle" ${ }^{\prime 2}$ ) forms the medial limit of the native ACL footprint. ${ }^{7,21,27}$ The center of the footprint is located $5.7 \pm 1.1 \mathrm{~mm}$ before $^{7}$ and $5.0 \pm 1 \mathrm{~mm}$ lateral to the medial tibial spine. ${ }^{27}$ Both of these structures are easily visible during arthroscopy and are, in our opinion, reliable landmarks for AP but also ML positioning.

The retro-eminence ridge (also called "over-the-back ridge" $^{\prime 25,27}$ or "eminentia intercondylaris" $"$ ), converging point between the medial and the lateral intercondylar ridge is sometimes considered the posterior limit of the ACL footprint. ${ }^{9,21}$ But the studies of Hara et al. ${ }^{28}$ and Tensho et al. ${ }^{20}$ tend to prove that there is a ligamentfree zone behind the ACL footprint, located right before the retro-eminence ridge corresponding to the intertubercular fossa.

In this study we chose to use the posterior aspect of the intertubercular fossa rather than the retroeminence ridge as the posterior limit of our grid, since the exact summit of the ridge was difficult to evaluate precisely on axial views on the CT scan. Additional studies are necessary to assess the anatomic relationship between the retro-eminence ridge and the intertubercular fossa to be able to localize it precisely during arthroscopy.

The retro-eminence ridge, located right in front of the posterior cruciate ligament (PCL) tibial insertion, can be palpated with a probe during an arthroscopic procedure. The posterior margin of the tibial footprint and the center of the footprint are located $10^{27,29}$ and 13 to $15^{27,30} \mathrm{~mm}$, respectively before it. The anterior ridge, or Parson's knob, a bony prominence located at the anterior aspect of the intercondylar area, is a common insertion of the anterior horn of the medial meniscus

Table 3. Comparison of position (in millimeters) between the center of tunnel and the center of the footprint according to intercondylar area method

\begin{tabular}{lccc}
\hline & Mean & $\begin{array}{c}\text { Standard } \\
\text { Deviation }\end{array}$ & Range \\
\hline Grid AP length & 29.5 & 3.0 & 16.2 to 36.7 \\
Grid ML length & 9.4 & 2.1 & 3.3 to 13.8 \\
Center of tunnel, AP & 8.8 & 3.2 & 1 to 17.2 \\
Center of tunnel, ML & 3.4 & 1.8 & -2.6 to 7.4 \\
Center of footprint, PA & 10.1 & 1.5 & 6.2 to 13.8 \\
Center of footprint, ML & 4.1 & 0.63 & 2.5 to 5.9 \\
Tunnel AP gap & -1.32 & 2.7 & -9.4 to 7 \\
Tunnels ML gap & -0.66 & 1.56 & -6.8 to 2.3 \\
\hline
\end{tabular}

Distances measured from posterior to anterior and from medial to lateral.

Abbreviations: AP, anteroposterior; ML, mediolateral. 
Table 4. Accuracy of the tunnel positioning relative to the anterior cruciate ligament footprint according to both methods

\begin{tabular}{|c|c|c|c|c|c|c|}
\hline & \multicolumn{2}{|c|}{ Anteroposterior } & \multicolumn{2}{|c|}{ Mediolateral } & \multicolumn{2}{|c|}{ Anteroposterior and Mediolateral } \\
\hline & $\mathrm{n}$ & $\%$ & $\mathrm{n}$ & $\%$ & $\mathrm{n}$ & $\%$ \\
\hline \multicolumn{7}{|l|}{ Not correct $(>2 \mathrm{~mm})$} \\
\hline Intercondylar area & 59 & 49.2 & 23 & 19.2 & 73 & 60.8 \\
\hline \multicolumn{7}{|l|}{ Correct $(<2 \mathrm{~mm})$} \\
\hline Reference standard & 77 & 64.2 & 104 & 86.7 & 66 & 55.0 \\
\hline Intercondylar area & 61 & 50.8 & 97 & 80.8 & 47 & 39.2 \\
\hline Total & 120 & 100.0 & 120 & 100.0 & 120 & 100.0 \\
\hline
\end{tabular}

and of the most anteromedial fibers of the ACL. It is a reliable arthroscopic landmark..$^{20,31,32}$

The intermeniscal ligament (transverse ligament) coincides with the most anterior edge of the tibial footprint. ${ }^{7,8}$ The center of the footprint is located 9.1 $\pm 1.5 \mathrm{~mm}$ behind it. ${ }^{7}$ Its arthroscopic visualization is inconstant but facilitated by experience and a axial (bird's-eye) view of the intercondylar area. ${ }^{33}$

The use of the anterior root of the lateral meniscus is controversial. According to Ziegler et al., ${ }^{30}$ the center of the tunnel must be located $7.5 \mathrm{~mm}$ medially and 10.2 $\mathrm{mm}$ behind the anterior aspect of it. However, Ferreti et al. ${ }^{7}$ and Hutchinson and Bae $^{34}$ did not find any constant correlation between the center of the footprint and the anterior and the posterior aspect of it. Tensho et al. $^{20}$ described the anterior horn of the lateral meniscus as a reliable landmark for the lateral limit of the footprint suitable for tunnel placement. The anterior root of the medial meniscus is constantly anterior to the ACL footprint. ${ }^{7}$

The PCL is also described as a landmark. In a cadaveric study, Hutchinson et al. ${ }^{34}$ found that the anterior margin of the PCL was 10.9 posterior to the center of the footprint in the sagittal plane. This is not consistent with previously cited findings concerning the retroeminence ridge. Moreover, its use is unreliable because it is covered with fat and synovium, whose thickness is not assessable. ${ }^{9,27}$

In summary, arthroscopic landmarks must be easily visible and constant and must allow reproducible placement of the tibial tunnel. The most reliable landmarks seem to be the bony ones in the intercondylar area, since they are visible or palpable. They also have the advantage to be visible on 3D CT scans and to allow the surgeon to easily compare arthroscopic positioning and the 3D control. Soft tissue landmarks, such as the ACL remnant, can also be used, but the surgeon must be aware of their limits and should combine their use with bony landmarks. Some recent surgical techniques are based on this fine anatomy of the intercondylar area. $^{35,36}$

\section{Limitations}

There are several limitations in our study. Singleobserver and single measurements did not allow for assessment of inter- or intra-observer reliability. In addition, tunnel severe malpositioning makes use of the intercondylar method difficult, because of destruction of the normal anatomic landmarks. In such cases, positions had to be extrapolated from the remaining anatomy of the intercondylar area. Moreover, the ovalshaped tunnel that we drill during ACL reconstruction does not correspond necessarily to a native ACL footprint, since $22 \%$ to $50 \%$ of them are triangular with an anterior base. There may be a mistake in the postulate that the aimed position for the center of the tunnel is the center of the ACL footprint. ${ }^{7,37}$ In addition, we do not practice double-bundle reconstructions, for which most of the studies cited in the discussion were made. Finally, this is a retrospective study, and we did not evaluate clinical results in combination with our radiological findings.

\section{Conclusion}

This intercondylar area method using arthroscopic landmarks can be used to assess tunnel placement on 3D CT scans after ACL reconstruction.

\section{References}

1. Hussein M, van Eck CF, Cretnik A, Dinevski D, Fu FH. Prospective randomized clinical evaluation of conventional single-bundle, anatomic single-bundle, and anatomic double-bundle anterior cruciate ligament reconstruction: 281 cases with 3- to 5-year follow-up. Am J Sports Med 2012;40:512-520.

2. Musahl V, Plakseychuk A, VanScyoc A, et al. Varying femoral tunnels between the anatomical footprint and isometric positions: Effect on kinematics of the anterior cruciate ligament-reconstructed knee. Am J Sports Med 2005;33:712-718.

3. Kim HS, Seon JK, Jo AR. Current trends in anterior cruciate ligament reconstruction. Knee Surg Relat Res 2013;25: 165-173. 
4. Fu FH, Van Eck CF, Tashman S, Irrgang JJ, Moreland MS. Anatomic anterior cruciate ligament reconstruction: A changing paradigm. Knee Surg Sorts Traumatol Arthrosc 2015;23:640-648.

5. Middleton KK, Hamilton T, Irrgang JJ, Karlsson J, Harner CD, Fu FH. Anatomic anterior cruciate ligament (ACL) reconstruction: A global perspective. Part 1. Knee Surg Sports Traumatol Arthrosc 2014;22:1467-1482.

6. Steiner ME, Battaglia TC, Heming JF, Rand JD, Festa A, Baria M. Independent drilling outperforms conventional transtibial drilling in anterior cruciate ligament reconstruction. Am J Sports Med 2009;37:1912-1919.

7. Ferretti M, Doca D, Ingham SM, Cohen M, Fu FH. Bony and soft tissue landmarks of the ACL tibial insertion site: An anatomical study. Knee Surg Sports Traumatol Arthrose 2012;20:62-68.

8. Kongcharoensombat W, Ochi M, Abouheif M, et al. The transverse ligament as a landmark for tibial sagittal insertions of the anterior cruciate ligament: A cadaveric study. Arthroscopy $2011 ; 27: 1395-1399$.

9. Siebold R, Ellert T, Metz S, Metz J. Tibial insertions of the anteromedial and posterolateral bundles of the anterior cruciate ligament: Morphometry, arthroscopic landmarks, and orientation model for bone tunnel placement. Arthroscopy 2008;24:154-161.

10. Parkinson B, Gogna R, Robb C, Thompson P, Spalding T. Anatomic ACL reconstruction: The normal central tibial footprint position and a standardised technique for measuring tibial tunnel location on 3D CT. Knee Surg Sports Traumatol Arthrosc 2017;25:1568-1575.

11. Han Y, Kurzencwyg D, Hart A, Powell T, Martineau PA. Measuring the anterior cruciate ligament's footprints by three-dimensional magnetic resonance imaging. Knee Surg Sports Traumatol Arthrosc 2012;20:986-995.

12. Sirleo L, Innocenti M, Innocenti M, Civinini R, Carulli C, Matassi F. Post-operative 3D CT feedback improves accuracy and precision in the learning curve of anatomic ACL femoral tunnel placement. Knee Surg Sports Traumatol Arthrosc 2018;26:468-477.

13. Parkar AP, Adriaensen MEAPM, Fischer-Bredenbeck C, et al. Measurements of tunnel placements after anterior cruciate ligament reconstruction: A comparison between CT, radiographs and MRI. Knee 2015;22:574-579.

14. Amis AA, Jakob RP. Anterior cruciate ligament graft positioning, tensioning and twisting. Knee Surg Sports Traumatol Arthrosc 1998;6:S2-S12 (suppl 1).

15. Stäubli HU, Rauschning W. Tibial attachment area of the anterior cruciate ligament in the extended knee position: Anatomy and cryosections in vitro complemented by magnetic resonance arthrography in vivo. Knee Surg Sports Traumatol Arthrosc 1994;2:138-146.

16. Edwards A, Bull AMJ, Amis AA. The attachments of the anteromedial and posterolateral fibre bundles of the anterior cruciate ligament. Part 2: Femoral attachment. Knee Surg Sports Traumatol Arthrosc 2008;16:29-36.

17. Bernard M, Hertel P, Hornung H, Cierpinski T. Femoral insertion of the ACL. Radiographic quadrant method. Am J Knee Surg 1997;10:14-21-2.

18. Lertwanich P, Martins CAQ, Asai S, Ingham SJM, Smolinski P, Fu FH. Anterior cruciate ligament tunnel position measurement reliability on 3-dimensional reconstructed computed tomography. Arthroscopy 2011:27:391-398.

19. Bernard M, Hertel P, Hornung H, Cierpinski T. Femoral insertion of the ACL. Radiographic quadrant method. Am J Knee Surg 1997;10:12-14.

20. Tensho K, Shimodaira H, Aoki T, et al. Bony landmarks of the anterior cruciate ligament tibial footprint: A detailed analysis comparing 3-dimensional computed tomography images to visual and histological evaluations. Am J Sports Med 2014;42:1433-1440.

21. Purnell ML, Larson AI, Clancy W. Anterior cruciate ligament insertions on the tibia and femur and their relationships to critical bony landmarks using highresolution volume-rendering computed tomography. Am J Sports Med 2008;36:2083-2090.

22. Sadoghi P, Borbas P, Friesenbichler J, et al. Evaluating the tibial and femoral insertion site of the anterior cruciate ligament using an objective coordinate system: A cadaver study. Injury 2012;43:1771-1775.

23. Kopf S, Musahl V, Tashman S, Szczodry M, Shen W, $\mathrm{Fu}$ FH. A systematic review of the femoral origin and tibial insertion morphology of the ACL. Knee Surg Sports Traumatol Arthrosc 2009;17:213-219.

24. Hoshino Y, Kim D, Fu FH. Three-dimensional anatomic evaluation of the anterior cruciate ligament for planning reconstruction. Anat Res Int 2012;2012:569704.

25. McGuire DA, Hendricks SD, Sanders HM. The relationship between anterior cruciate ligament reconstruction tibial tunnel location and the anterior aspect of the posterior cruciate ligament insertion. Arthroscopy 1997;13:465-473.

26. Grasso S, Linklater J, Li Q, Parker DA. Validation of an MRI protocol for routine quantitative assessment of tunnel position in anterior cruciate ligament reconstruction. Am J Sports Med 2018;46:1624-1631.

27. Edwards A, Bull AMJ, Amis AA. The attachments of the anteromedial and posterolateral fibre bundles of the anterior cruciate ligament: Part 1: Tibial attachment. Knee Surg Sports Traumatol Arthrosc 2008;16:29-36.

28. Hara K, Mochizuki T, Sekiya I, Yamaguchi K, Akita K, Muneta T. Anatomy of normal human anterior cruciate ligament attachments evaluated by divided small bundles. Am J Sports Med 2009;37:2386-2391.

29. Colombet P, Robinson J, Christel P, et al. Morphology of anterior cruciate ligament attachments for anatomic reconstruction: A cadaveric dissection and radiographic study. Arthroscopy 2006;22:984-992.

30. Ziegler CG, Pietrini SD, Westerhaus BD, et al. Arthroscopically pertinent landmarks for tunnel positioning in single-bundle and double-bundle anterior cruciate ligament reconstructions. Am J Sports Med 2011;39: $743-752$.

31. Berg EE. Parsons' knob (tuberculum intercondylare tertium). A guide to tibial anterior cruciate ligament insertion. Clin Orthop Relat Res 1993;(292):229-231.

32. Shimodaira H, Tensho K, Akaoka Y, Takanashi S, Kato H, Saito N. Remnant-preserving tibial tunnel positioning using anatomic landmarks in double-bundle anterior cruciate ligament reconstruction. Arthroscopy 2015;32: 1822-1830.

33. Crain EH, Fithian DC, Paxton EW, Luetzow WF. Variation in anterior cruciate ligament scar pattern: Does the scar 
pattern affect anterior laxity in anterior cruciate ligamentdeficient knees? Arthroscopy 2005;21:19-24.

34. Hutchinson MR, Bae TS. Reproducibility of anatomic tibial landmarks for anterior cruciate ligament reconstructions. Am J Sports Med 2001;29:777-780.

35. Shimodaira H, Tensho K, Akaoka Y, Takanashi S, Kato H, Saito N. Tibial tunnel positioning technique using bony/ anatomical landmarks in anatomical anterior cruciate ligament reconstruction. Arthrosc Tech 2017;6:e49-e55.
36. Buscayret F, Temponi EF, Saithna A, Thaunat M, Sonnery-Cottet B. Three-dimensional CT evaluation of tunnel positioning in ACL reconstruction using the single anteromedial bundle biological augmentation (SAMBBA) technique. Orthop J Sport Med 2017;5:1-7.

37. Tallay A, Lim M-H, Bartlett J. Anatomical study of the human anterior cruciate ligament stump's tibial insertion footprint. Knee Surg Sports Traumatol Arthrosc 2008;16: $741-746$. 